\title{
CALIDAD EDUCATIVA: UNA MIRADA DESDE SUS ACTORES ${ }^{20}$ QUALIDADE EDUCACIONAL: UM OLHAR DO SEUS ATORES EDUCATIONAL QUALITY: A LOOK FROM ITS ACTORS
}

\author{
Sandra C. Buitrago $\mathrm{R}^{21}$ \\ Maria Virginia M. Dazzani ${ }^{22}$ \\ Ingrid A. Buitrago Rivera ${ }^{23}$ \\ Giovana Zen ${ }^{24}$
}

\begin{abstract}
Resumen
La presente investigación aborda los significados sobre calidad educativa a partir de la perspectiva simbólica de un grupo de profesores universitarios, desde el enfoque sociocultural Vigotskyano. En ella se prioriza al docente como sujeto de pesquisa, comprendiendo cómo el profesor universitario en Colombia define la calidad educativa y la analiza a partir del contexto de su universidad. Para tal efecto, se entrevistan dos grupos de profesores (de una universidad pública y una privada), además, de un grupo focal para cada tipo de universidad. A partir de los hallazgos de ésta, se aportan elementos para la re-significación de la calidad educativa trascendiendo los preconceptos teóricos establecidos a lo largo de los tiempos.
\end{abstract}

Palabras claves: Calidad educativa. Educación Superior. Significados. Socio-cultural.

\section{Resumo}

A presente pesquisa aborda os significados da qualidade educacional sob a perspectiva simbólica de um grupo de professores universitários, a partir da abordagem sociocultural Vygotskiana. Nele, o professor é priorizado como sujeito de pesquisa, entendendo como o professor universitário na Colômbia define a qualidade da educação e a analisa a partir do contexto de sua universidade. Para esse fim, dois grupos de professores (de uma universidade pública e uma privada) são entrevistados, bem como um grupo focal para cada tipo de universidade. A partir dos resultados deste, elementos para re-significação da qualidade da educação fornecem transcender os preconceitos teóricos estabelecidos ao longo do tempo.

\footnotetext{
${ }^{20}$ Parte da tese de doutorado em Educação financiado pela Coordenação de aperfeiçoamento de pessoal de nível superior (CAPES). Texto aprovado pelo Comitê de Ética em Pesquisa, CAAE: 75501317.7.0000.5531

${ }^{21}$ Maestría en Educación en la Universidad Santo Tomas, Bogotá, Colombia. Doctoranda en educación en la Universidad Federal de Bahía, UFBA, Brasil. Bolsista CAPES. sandra.carolina@ufba.br

${ }^{22}$ Doctora en educación. Profesora Asociada a la Universidad Federal da Bahía, Salvador, Brasil. Investigadora del Programa de Posgrado em Psicología y Educación. dazzani@ufba.br

${ }^{23}$ Maestría en Educación en la Universidad Federal Fluminense (UFF). Miembro del Grupo de Investigación Políticas em Educação: Formação, Cultura e Inclusão. andreabuitragorivera@gmail.com

${ }^{24}$ Doctorado en Educación en la Universidad Federal de Bahía (UFBA). Profesora Adjunta de la facultad de Educación de la Universidad Federal de Bahía. giovanacristinazen@gmail.com
} 


\title{
RevistAleph
}

Palavras-chaves: Qualidade educacional. Educação superior. Significados.

Sociocultural.

\begin{abstract}
The present research addresses the meanings of educational quality from the symbolic perspective of a group of university professors, from the Vygotskian socio-cultural approach. In it, the teacher is prioritized as a research subject, understanding how the university professor in Colombia defines educational quality and analyzes it from the context of his/her university. For this purpose, a group of professors from a public and a private university are interviewed, as well as a focus group for each type of university. Based on the findings of this, elements for the re-signification of educational quality are transcended, transcending the theoretical preconceptions established over time.
\end{abstract}

Keywords: Educational quality. Higher education. Meanings. Socio-cultural.

\section{Introducción}

El presente artículo indaga los significados que los profesores universitarios construyen sobre la calidad educativa, pues últimamente una de las palabras más utilizadas sobre todo en el ámbito educativo es calidad. Se habla de calidad de vida, calidad de las instituciones y calidad en el trabajo, la mayoría de las veces sin precisar qué se entiende por calidad o desde qué enfoque se utiliza este término, o como lo menciona Peirce (1984) en su teoría semiótica, la comunidad en general les asigna a sus mundos simbólicos diferentes significados y maneras de actuar. La variedad de teorías y definiciones que se formulan sobre calidad educativa dependen lógicamente de los intereses, experiencias y perspectivas que se asumen en cada caso, lo que ocasiona una evidente falta de consenso. Se puede decir que el término calidad es un concepto relativo, que puede ser definido desde una perspectiva multidimensional, y al mismo tiempo subjetivo pues va a depender mucho de la cultura en que éste se desenvuelve (GIRALDO, ABAD e DÍAZ, 2005).

La insuficiente teoría sobre calidad y el atolladero para poder llegar a un consenso entre las distintas audiencias implicadas, impiden determinar una definición de este concepto que sea aceptada de forma unánime (DE MIGUEL DIAZ, 1995). Se sabe que todos los signos que rodean al mundo varían en términos de calidad y al mismo tiempo todos difieren en su apreciación. Aunque se viven momentos en los que la 


\section{RevistAleph}

"cultura de la calidad" constituye algo más que una simple moda, se debe reconocer que su significado es subjetivo para cada interpretante, al igual que para otros conceptos como por ejemplo el de belleza, por lo que es difícil efectuar una definición satisfactoria para todos (DE MIGUEL DIAZ, 1995).

El debate sobre los desafíos de la calidad han sido siempre un reto en la profesión académica, pues se reconoce que la educación se ha convertido en una importante fuente fértil de producción de conocimientos, de ahí que se les hayan asignado nuevas tareas a las instituciones educativas y en particular a la universidad, por su creciente responsabilidad en formar la fuerza más calificada de la sociedad.

De modo que, se identifica el papel del docente como el actor principal en quien recae la calidad educativa que plantea el Ministerio colombiano de Educación Nacional (MEN); sin embargo, gran parte de estos docentes no están dotados de los mecanismos y condiciones necesarias para ejercer esta labor, en primer instancia porque las políticas contemplan solo la idea exógena de la labor docente profesor (evaluaciones, rutas de mejoramiento, caracterización institucional, auditorias, visitas, certificación, etc.) y se abandona por completo la idea endógena del profesor (sentimientos, presiones, relaciones interpersonales, significados, perspectiva simbólica, incentivos socio-afectivos, formación profesional y personal, etc.), por lo que surge la siguiente pregunta de investigación ¿cuáles son los significados sobre calidad educativa que tienen un grupo de profesores universitarios en Colombia?.

Para ello se considera pertinente indagar esta situación desde la interpretación que hacen los docentes sobre el significado de calidad educativa, quienes son los actores primarios de este contexto. Analizando también la influencia del significado con la realidad inmersa en la que se encuentra la población objeto (universidad), cuya finalidad permita conocer la incidencia que tiene el significado dentro de una determinada cultura académica bajo el abordaje de las corrientes literarias trabajadas para la presente investigación. Profundizando especialmente en las categorías teóricas de significados según Peirce (1984); la psicología cultural desde la perspectiva semiótica de Jaan Valsiner trabajada en su más reciente libro "An Invitation to Cultural Psychology" (2014), y el enfoque socio-cultural de Vygotsky. 


\section{RevistAleph}

Los trabajos de Charles Peirce, constituyen el aporte más característico a la teoría de la significación. Peirce plantea que los signos pueden ser arbitrarios en el comienzo (principalmente signos de las lenguas humanas) componiendo solo una parte de la esfera total de los signos (los lenguajes) (MERRELL, 2012). El concepto Peirciano de signo consiste de un representamen (llamado con frecuencia signo) que se relaciona con un objeto (que puede ser un acto o acontecimiento) para que sea de hecho un signo, también se relaciona con un tercer término al que Peirce llama de interpretante (que es el responsable por engendrar el significado de signo) (MERRELL, 2012). El modelo más fundamental de la tricotomía de Peirce está compuesto por íconos, índices y símbolos. Los iconos se parecen a los objetos con los cuales se relaciona, lo índices se relacionan con sus objetos por alguna conexión natural, y la relación entre los símbolos y sus objetos se da por el uso habitual del signo y de acuerdo con una convención cultural. Los símbolos más ejemplares y comunes son aquellos del lenguaje.

Por su parte, Valsiner (2014) fundamentándose en elementos de la semiótica de Peirce, por ejemplo, su conocida concepción tríadica de signo, objeto e interpretante, desarrolla sus reflexiones abarcando, entre otras temáticas, las cargas de afectividad implícitas en las mediaciones semióticas humanas; la cognición inconsciente y la modularidad de la mente, la construcción semiótica que entrelaza pasado, presente y futuro, y que contribuye a la génesis de la subjetividad en su relación con la intersubjetividad y su vínculo ineludible con la vida inter-psicológica de la cultura. Así, la psicología cultural se propone estudiar las variadísimas maneras en las que las tradiciones culturales y las prácticas sociales regulan, expresan, transforman y transmutan la psique humana (VALSINER, 2014). Es el estudio de las formas como el sujeto se constituye en una permanente interrelación con el otro, en el marco de los sistemas culturales que rigen las prácticas sociales en un momento histórico dado. La psique no se da sino en una cultura particular y es esa cultura la que confiere su particularidad, considerando la cultura como un sistema de conocimiento que proporciona un modelo de realidad, a través del cual se da sentido a nuestro comportamiento. 


\section{RevistAleph}

A partir de lo anterior, es como se plantea la conciencia del significado del concepto desde sus actores para construir significados alternos al término calidad educativa y darle otros sentidos a su práctica; y se discute el rol que los docentes tienen para desempeñar la reflexión y la acción para el logro de esta meta, desde el enfoque socio cultural Vigotskyano, ya que en la mayoría de los discursos y revisiones teóricas que argumentan este concepto lo hacen desde una lógica empresarial. Una de las tesis centrales del enfoque socio cultural es que la conciencia se forma y se transforma en el curso de actividades humanas. De acuerdo con Rodriguez (2010, p 3), "Estas actividades, que se realizan socialmente, se caracterizan por estar mediadas por herramientas y signos que son productos de la cultura y por estar históricamente situadas". Estos significados se convierten en el conjunto de herramientas que se usan para construir nuestra realidad y actuar sobre ella. Una vez que el sujeto se apropia de ellos, estos significados comienzan a mediar su pensamiento y su forma de solucionar problemas (Karpov, 2003), por ello, la comprensión de éstos es una condición necesaria para una práctica reflexiva, critica y emancipadora.

Sobre la base de lo expuesto, es relevante para esta investigación en curso, abordar la perspectiva del concepto de calidad educativa desde la reflexión sociocultural y del sentido endógeno del docente de educación superior. Así, el enfoque socio-cultural tiene como premisa fundamental la idea de que para comprender la conciencia humana es necesario conocer las formas de actividad en las que se constituye, los instrumentos (concretos y simbólicos) con los que realiza dicha actividad, y las formas sociales en que se aprende el uso de esos instrumentos y su valor para transformar el medio ambiente y las condiciones de vida (RODRÍGUEZ, 2010), lo que quiere decir, que la percepción o significados de las personas entorno a algún hecho o fenómeno es producto de la vida en sociedad, a través de los signos y símbolos compartidos para realizar las actividades que dan sentido a su realidad. Para Rodríguez (2010), la perspectiva socio-cultural no sólo establece el significado de la palabra como unidad de análisis para el estudio del pensamiento, sino que establece la elaboración de sentido subjetivo como unidad de análisis para la comprensión de la acción humana al considerar los motivos, la afectividad y la emotividad implicados en los actos de pensamiento. 


\section{RevistAleph}

El enfoque socio-cultural encamina a mirar más allá de lo evidente, a situar las acciones humanas en su contexto histórico y a examinar las condiciones que llevan a la producción de conocimientos. Sólo desde una óptica que presuponga lo dicho como esencia de las producciones humanas, se puede interrogar el significado de las palabras y las representaciones de la realidad que se expresan en ideas y conceptos.

Por otro lado, también es importante reconocer que la literatura sobre el tema de calidad se ha direccionado hacia el análisis, estipulación y relación de indicadores de calidad que establecen diferencias entre los niveles iniciales, intermedios, superiores y universitarios. No obstante, no se puede inferir un solo significado, aún más si ese significado parece enajenarse de la dimensión socio-cultural del concepto y lo presenta como una categoría natural, acabada e indiscutible.

Ante este panorama, es pertinente problematizar el concepto de calidad educativa, analizar críticamente los supuestos en que se sostiene y los procedimientos que se utilizan para darle validez, y proponer significaciones alternas. Es así como en este proceso, la perspectiva socio-cultural ofrece herramientas conceptuales y metodológicas para realizar esta importante tarea (RODRÍGUEZ, 2010).

\section{Metodología}

El diseño metodológico se fundamenta desde el método cualitativo bajo el enfoque socio-cultural o también llamado histórico-cultural de Vygotsky, relacionado con la antropología y la psicología social.

En este orden de ideas, se parte de que la presente investigación es empírica puesto que Cerda (2013) considera la experiencia sensorial como única fuente del saber, afirmando que todo conocimiento se fundamenta en la experiencia y se adquiere a través de la misma. Asimismo, aborda como eje fundamental el estudio de caso, porque comprende en profundidad la realidad social y educativa cuyo propósito es establecer un marco de trabajo para la discusión y debate entre docentes. La modalidad de estudio será estudio de caso intrínseco que, según Stake (1998), son casos con especificidades propias, que tienen un valor en sí mismos y pretenden alcanzar una mejor comprensión 


\section{RevistAleph}

del caso concreto a estudiar. En este supuesto no se elige al caso porque sea representativo de otros casos, o porque ilustre un determinado problema o rasgo, sino porque el caso en sí es de interés.

La recolección de datos se lleva a cabo a través de grupos focales y entrevistas semiestructuradas. Los grupos focales permiten al investigador obtener una noción general del grupo a abordar y conocer las opiniones o actitudes mediante la interacción del grupo en una dinámica en que los participantes se sienten cómodos y libres de hablar y comentar sus percepciones. Según Sampieri (2014), en los grupos de enfoque existe un interés por parte del investigador por cómo los individuos forman un esquema o perspectiva de un problema; algunos autores los consideran como una especie de entrevistas grupales que consisten en reuniones de grupos pequeños o medianos (tres a diez personas), en las cuales los participantes conversan a profundidad en torno a uno o varios temas a través de la interacción, donde el objetivo es generar y analizar la interacción entre ellos y cómo se construyen grupalmente significados. Creswell (2005) indica que el tamaño de los grupos varía dependiendo del tema: en esta investigación se trabaja con cuatro docentes porque se aborda una temática multi interpretativa y subjetiva donde se requiere de la visión especifica de esta población. Para Sampieri (2014), el grupo focal es una técnica de recolección de datos, la unidad de análisis es el grupo (lo que expresa y construye) y tiene su origen en las dinámicas grupales, muy socorridas en la psicología. El formato de las sesiones consiste en reunir un grupo de personas para trabajar con los significados, conceptos, las experiencias, emociones, creencias, categorías, sucesos o los temas que interesan en el planteamiento de la presente investigación. Basándose en la teoría de Sampieri (2014), se siguen los siguientes pasos para realizar el grupo focal:

1. Se determina según el desarrollo de la investigación, dos sesiones del grupo focal: una sesión con el grupo de profesores de la universidad privada y otra sesión con el grupo de profesores de la universidad pública, cada una compuesta por cuatro docentes. En tal caso, no se pueden fusionar los dos 


\section{RevistAleph}

grupos por dificultades geográficas ya que cada uno se encuentra en diferentes ciudades.

2. El perfil de los individuos son docentes de tiempo completo con experiencia mínima laboral en la institución de educación superior de cuatro años y nivel de formación (especialista y/o magister).

3. Se organiza la sesión en un lugar confortable, silencioso y aislado. Los participantes deben sentirse tranquilos y relajados.

4. Se elabora el reporte de la sesión, el cual incluye principalmente:

- Datos sobre los participantes (edad, género, nivel educativo y todo aquello que es relevante para el estudio).

- Fecha y duración de la sesión (hora de inicio y terminación).

- Grabación en audio.

En un segundo momento se aplican entrevistas semiestructuradas que permiten más profundidad y aclarar cualquier duda o inquietud generada en el grupo focal, facilitando identificar el conocimiento del entrevistado, determinar mejor qué piensa realmente el entrevistado, respuestas no esperadas o apreciaciones adicionales no comentadas en el grupo focal; por ello, permite una mayor libertad y flexibilidad en la obtención de información. La entrevista se define como una reunión para conversar e intercambiar información entre una persona (el entrevistador) y otra (el entrevistado) u otras (entrevistados). En la entrevista, a través de las preguntas y respuestas se logra una comunicación y la construcción conjunta de significados respecto a un tema (JANESICK, 1998).

Después, se llevarán a cabo en un segundo momento entrevistas semiestructuradas a cuatro profesores, basadas en una guía de preguntas en la que el entrevistador tiene la libertad de introducir preguntas adicionales para precisar conceptos; estas se usan comúnmente como herramientas para recolectar datos cualitativos y cuando el problema de estudio no se puede observar o es muy difícil hacerlo por ética o complejidad y obtener mayor información (SAMPIERI, 2014). 


\section{RevistAleph}

\section{Resultados y Discusiones iniciales}

La pertinencia de la postura teórica elegida permitió comprender y analizar los significados que construyen los profesores universitarios sobre el tema de calidad educativa. Nos encamina a mirar más allá de lo que aparentemente es "evidente", a situar las acciones humanas en su contexto histórico, y a examinar las condiciones que llevan a los profesores a la construcción de dichos significados.

Tabla 1 - Esquema de análisis de los significados detectados en el discurso de los grupos focales y las entrevistas semiestructuradas de la universidad privada y de la universidad pública

\begin{tabular}{c|l}
\hline Objetivo & \multicolumn{1}{c}{ Algunos significados mencionados } \\
\hline OB3 & - Educación \\
& - Reivindicación de la labor docente/ empoderamiento de su conocimiento \\
& y campo profesional \\
& - El profesor universitario debe ser un transformador de vidas, empático y \\
& mediador \\
\hline OB1 & - Calidad educativa \\
& - Dar respuestas a criterios del MEN/ pruebas estandarizadas \\
\hline OB2 & - Singularidades del sector público y privado \\
& seconocimiento social de los maestros. Dar garantías donde el docente \\
& incluso en su proceso de gestión \\
\hline OB4 & - Ausencia de las humanidades en los currículos \\
& Proyectos de nación que reconozca a los seres humanos como ciudadanos \\
& Políticas públicas de calidad
\end{tabular}

Fuente: Creación propia

Dentro de este contexto, el significado de calidad solo se puede repensar desde su realidad, su puesta en práctica, para transformar su significación y construir un sentido nuevo. Por ello, comprender el origen y significado del concepto, sus acepciones y las prácticas que lo convierte en actividad que regula la educación, son una condición necesaria para la transformación de la realidad que ha conformado. De modo que, la concepción de la calidad de educación tiene una profunda connotación social, ideológica y política enmarcada por el contexto de la región en el que los profesores se desenvuelven. 


\section{RevistAleph}

En síntesis, la calidad tendría que estar asociada con las respuestas que dé la academia, que dé el sistema educativo, frente a las necesidades del país, aportando soluciones que impacten al país. De modo que existe la urgencia de empoderar más a los profesores que a la burocracia, esto supone que la autonomía que se les otorgue ha de ser el motor que los impulse a diseñar, dinamizar y gestionar una mejor calidad educativa. Desde esta perspectiva, el discurso de estos profesores, es en conjunto un proceso de empoderamiento, es decir, el camino por el cual ellos se convierten en actores primordiales a través de organizarse, de imaginar lo que sería para ellos el ideal de calidad educativa, de discutir y trabajar por este objetivo, de tomar decisiones, de autogestionar su propio proceso de mejora y eliminar prácticas y políticas contradictorias.

En términos generales, el problema de la calidad no reside en los docentes, sino en el sistema con el que opera la universidad. Sin embargo, también se ve con toda claridad cómo la solución sí depende gran parte del equipo de docentes, siempre y cuando este equipo sea capaz de modificar el sistema. Entonces, mejorar la calidad de la educación significa orientar los esfuerzos hacia las personas.

\section{Conclusiones}

Es un hecho que en la actualidad los entes encargados de la regulación, evaluación y seguimiento de la calidad, aún no se toman en serio el concepto de calidad educativa en la educación superior, prevaleciendo primero los intereses personales de las organizaciones mercantiles, que de la formación del ser humano como tal, de ese mismo modo, la educación se enfrenta a un sin número de exigencias del siglo XXI con condiciones laborales, institucionales y reglamentares del siglo XIX; es de este modo como la presente investigación en curso comprende la estrecha relación entre la experiencia profesional docente con su significado de calidad, analizando dichos procesos de construcción de significado y su incidencia en la práctica, estableciendo la repercusión que tiene el contexto educativo social y cultural en la percepción e ideales de los maestros. Por ende, se pretende dignificar el rol docente y a partir de los hallazgos 


\section{RevistAleph}

de ésta, aportar elementos para la re-significación de la calidad educativa trascendiendo los preconceptos teóricos establecidos a lo largo de los tiempos, focalizando la calidad de la educación desde la perspectiva endógena, especialmente a partir de la perspectiva del profesor.

Es importante aclarar que cuando se habla de significados, no se hace referencia a la teoría, sino a los saberes, creencias, valores que ayudan a prefigurar el hacer que un profesor realiza como docente, ya que se puede decir que, de acuerdo con como el educador signifique su práctica, es la forma como la realiza (VERGARA, 2005). No es posible que el profesor haga cosas que desconoce, que no están en su conciencia, porque los significados están asimilados a sus esquemas previos. A partir de un sistema teórico nuevo no es posible, de manera prescriptiva, configurar la práctica de manera distinta (SACRISTAN, 1988), es necesario entender la absoluta interdependencia y articulación entre significado, conocimiento, conciencia y mentalidad con acción y con el saber hacer del educador. Desde esta perspectiva, la práctica educativa "es una acción orientada, con sentido, donde el sujeto tiene un papel fundamental como agente, aunque insertado en la estructura social" (SACRISTAN, 1988, p. 35).

\section{Referencias}

CERDA, H. Los elementos de la investigación. tercera. ed. Bogotá: El buho LTDA, 2013.

CRESWELL, J. Diseño de investigación. Aproximaciones. Buenos Aires: Sage, v. III, 2005. p. 143-171.

DE MIGUEL DIAZ, F. M. La calidad de la educacion y las variables de proceso y de producto. Cuadernos de Sección, Medellin, p. 29-51, 1995.

DUQUE, P. C.; GARCÍA, P. S. ; LABAO, T. M. Nuevos retos de la profesion docente. II SEMINARIO INTERNACIONAL RELFIDO (Red Europea y Latinoamericana de Formación e Innovación Docente). Barcelona: FODIP, 2010.

GIRALDO, U.; ABAD, D.; DÍAZ, E. Consejo Nacional de Acreditación, 2005. Disponivel em: <http://www.mineducacion.gov.co/CNA/1741/articles-186502_doc_academico10.pdf>. JANESICK, V. "Stretching" exercises for qualitative researchers. Thousand Oaks: Sage, 1998. KARPOV, Y. Vygotsky's doctrine of scientific concepts: Its role for contemporary. In: KARPOV, Y. Vygotsky's educational theory in cultural context. New York: Cambridge University Press, 2003. p. 65-82. 


\section{RevistAleph}

MERRELL, F. Semiótica de Charles S. Peirce hoje. [S.I.]: Unijuí Esoterismo , 2012.

MINISTERIO DE EDUCACIÓN NACIONAL. Educación de calidad para la prosperidad, 2011. Disponivel em: <http://www.mineducacion.gov.co/1621/article-259478.html>.

RODRÍGUEZ, W. El concepto de calidad educativa: una mirada crítica desde el enfoque históricocultural. Actualidades Investigativas en Educación, v. 10, n. 1, p. 1-28, Abril 2010. ISSN 1409-4703.

SACRISTAN, J. El curriculum: una reflexión sobre la práctica. Madrid: Morata, 1988.

SAMPIERI, R. Metodología de la investigación. sexta. ed. Mexico D.F.: M.C. GRAW HILL Education, 2014.

STAKE. Investigación con estudio de casos segunda. Madrid: Morata, 1988.

VALSINER, J. An Invitation to Cultural Psychology. London: SAGE, 2014.

VERGARA, M. Significados de la práctica docente que tienen los profesores. REICE - Revista Electrónica Iberoamericana sobre Calidad, Eficacia y Cambio en Educación, v. 3, n. 1, p. 1-13, 2005. 